\section{Feedback Stabilization Over a First Order Moving Average Gaussian Noise Channel}

\author{
Richard H. Middleton, Alejandro J. Rojas, \\ James S. Freudenberg, and Julio H. Braslavsky
}

\begin{abstract}
Recent developments in information theory by Y.-H. Kim have established the feedback capacity of a first order moving average additive Gaussian noise channel. Separate developments in control theory have examined linear time invariant feedback control stabilization under signal to noise ratio (SNR) constraints, including colored noise channels. This note considers the particular case of a minimum phase plant with relative degree one and a single unstable pole at $z=\phi$ (with $|\phi|>1$ ) over a first order moving average Gaussian channel. SNR constrained stabilization in this case is possible precisely when the feedback capacity of the channel satisfies $C_{F B} \geq \log _{2}|\phi|$. Furthermore, using the results of Kim we show that there exist linear encoding and decoding schemes that achieve stabilization within the SNR constraint precisely when $C_{F B} \geq \log _{2}|\phi|$.
\end{abstract}

Index Terms-Channel capacity, colored noise, control over communications, signal to noise ratio (SNR).

\section{INTRODUCTION}

There has been growing interest in connections between feedback control theory and communication systems. In the feedback control area, this has resulted in a number of publications and special issues on this topic such as [2]. In [3], the authors consider the joint design of communication and control strategies, and use arguments from rate distortion theory to show that linear strategies are optimal for first order systems. LQG style control over a binary channel is considered in [4]. A related line of research considers signal to noise ratio (SNR) constrained feedback control systems, see for example [5]-[7]. This paradigm readily extends to consideration of Gaussian channels with memory, [8].

In a largely separate line of research, there have also been a number of studies of feedback issues in communication channels. One particular issue relates to the ability for feedback to increase the "capacity" of a communication channel with memory, e.g., [9].

Channel capacity has been precisely characterized in the famous Shannon result for an additive white Gaussian noise (AWGN) channel with noise variance $\sigma^{2}$ and transmitted average power constraint $\mathcal{P}_{\max }$ $[9$, p.241]

$$
C=\frac{1}{2} \log _{2}\left(\frac{1+\mathcal{P}_{\max }}{\sigma^{2}}\right)
$$

The particular form of capacity considered in [10], [11] is based on the $n$ block feedback capacity, $C_{n, F B}$ of the channel. This is an operational definition of capacity, for which "reliable" communication using

Manuscript received May 16, 2008; revised September 26, 2008. Current version published January 14, 2009. This work was supported by the Science Foundation of Ireland SFI 07/RPR/I177 Recommended by Associate Editor D. Liberzon.

R. Middleton is with the Hamilton Institute, The National University of Ireland Maynooth, Co Kildare, Ireland, (e-mail: richard.middleton@nuim.ie).

A. Rojas and J. Braslavsky are with the ARC Centre of Excellence for Complex Dynamic Systems and Control, The University of Newcastle, Callaghan NSW 2308, Australia (e-mail: alejandro.rojas@newcastle.edu.au; julio.braslavsky@newcastle.edu.au).

J. Freudenberg is with the Department of Electrical Engineering and Computer Science, The University of Michigan, Ann Arbor, MI 48109-2122 USA (e-mail: jfr@eecs.umich.edu).

Digital Object Identifier 10.1109/TAC.2008.2007183 $n$ transmissions may be performed. We follow the definition of feedback capacity $C_{F B}$ used in [10]

$$
C_{F B}=\lim _{n \rightarrow \infty} C_{n, F B} .
$$

Results such as (1) for the AWGN case have proven difficult to generalize to the case of a colored noise channel with feedback ${ }^{1}$. It is well known that in the case of an AWGN channel that feedback does not alter the capacity, while in the case of an additive Colored Gaussian noise (ACGN) channel, it is possible for feedback to improve channel capacity.

For the case of an AWGN channel, linear coding schemes were used as a means of achieving capacity in [12]. This scheme was applied to ACGN channels in [13] (for the case of an auto-regressive channel) and recently in a more general setting in [14]. A link between the coding schemes of [12], [13] and a feedback structure involving an unstable system have been provided in [14]. These results focus primarily on autoregressive noise coloring, and use the linear coding structure of [12] to provide a lower bound on the feedback channel capacity. The authors of [15] discuss Kalman-Bucy filtering in relation to feedback communication over Gaussian channels with memory.

The results in [10] allow the capacity with feedback over an ACGN channel to be computed as the limit of an optimization problem but general results establishing what this limit is have proven elusive. For an (MA1) Gaussian channel, [11] gives a precise characterization of the feedback capacity in terms of the average signal power restriction for unity noise variance. This result can be trivially generalized to a result for a given average signal power to noise power ratio.

Here our interest is in stabilization over an ACGN communication channel, though this is closely related to the converse question of linear feedback coding design for an ACGN communication channel. We show that the results on feedback capacity in [11] (and parallel results in [15]) and the SNR constrained stabilization results of [8] are linked for the case of an MA1 channel and relative degree one, minimum phase plant with a single unstable pole at $z=\phi$. In this case stabilization within an SNR constraint is possible precisely when the feedback capacity of the channel, $C_{F B}$ (as in (2)) satisfies

$$
C_{F B} \geq \log _{2}(|\phi|) \text {. }
$$

Moreover, if stabilization is possible, it can be achieved by a linear scheme. This result parallels a simplified form of the results of [16], [17], neither of which apply immediately to colored noise channels. Note that [18] use information theoretic techniques to derive necessary conditions for stabilizability applicable to a large class of communication channels. A preliminary version of the present results was presented in [1].

We commence our technical note with some preliminary mathematical definitions as well as defining the class of communication channel models, and feedback systems we consider. In Section II we give a minor modification of the main result in [11] for the feedback capacity of an MA1 ACGN channel. We then turn our attention in Section III to problems of SNR constrained control for linear systems, where the main results are established.

\section{A. Preliminaries}

We shall generally use $k \in \mathbb{Z}^{+}$as the discrete time index, and upper case letters, such as $S_{k}, R_{k}$, to denote elements of sequences $\left\{S_{k}\right\}$,

\footnotetext{
${ }^{1}$ In this context, feedback means that the transmitted signals are permitted to depend in a causal fashion on the received information.
} 


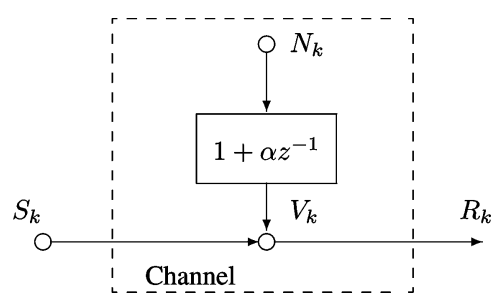

Fig. 1. First order moving average additive colored gaussian noise channel.

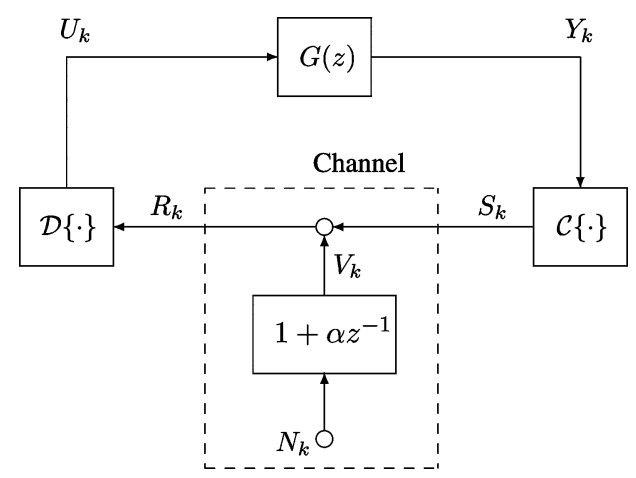

Fig. 2. Feedback stabilization over a communication channel.

$\left\{R_{k}\right\}$ of random variables. We use a superscript $k$ to denote a subsequence of random variables, for example $S^{k}:=\left\{S_{0}, S_{1}, \ldots, S_{k}\right\}$. The differential entropy of a random variable, e.g., $X_{k}$ is denoted by $h\left(X_{k}\right) . E[\bullet]$ is used for the expected value. Finite dimensional Linear Time Invariant (LTI) systems will be described by their rational transfer functions in the complex transform variable $z$.

By a slight abuse of notation, we shall use expressions such as $G(z) *$ $U_{k}$ to denote the convolution of the pulse response of $G(z)$ with $\left\{U_{k}\right\}$. We use $\mathcal{L}_{2}$ to denote the set of proper rational transfer functions with associated norm

$$
\|G(z)\|_{\mathcal{L}_{2}}^{2}=\frac{1}{2 \pi} \int_{-\pi}^{+\pi}\left|G\left(e^{j \theta}\right)\right|^{2} d \theta .
$$

$\mathcal{L}_{2}$ is therefore the space of rational proper transfer functions with no poles on the unit circle. We also define important subsets $\mathcal{H}_{2} \subset \mathcal{L}_{2}$ and its orthogonal complement $\mathcal{H}_{2}^{\perp} \subset \mathcal{L}_{2}$ as

$$
\begin{aligned}
\mathcal{H}_{2} & =\mathcal{L}_{2} \cap\{G(z): \text { analytic for }|z| \geq 1\} \\
\mathcal{H}_{2}^{\perp} & =\mathcal{L}_{2} \cap\{G(z): \text { analytic for }|z| \leq 1, G(0)=0\} .
\end{aligned}
$$

\section{B. The Channel Model}

The channel model we consider in this technical note is depicted in Fig. 1. The channel has input, output and noise denoted by $S_{k}, R_{k}$ and $V_{k}$ respectively.

Mathematical relationships for the communication channel in Fig. 2 are described below. First, the received signal $R_{k}$ is given by

$$
R_{k}=S_{k}+V_{k} \text {. }
$$

The channel noise, $V_{k}$ in (7), is generated by an MA1 process

$$
V_{k}=N_{k}+\alpha N_{k-1},
$$

where $N_{k}$ is an IID Gaussian process, with variance $\sigma^{2}$, and we assume that $|\alpha|<1$.
The channel transmission is required to satisfy an average power constraint $^{2}$

$$
\lim _{N \rightarrow \infty}\left\{\frac{1}{N} \sum_{k=1}^{N} E\left[S_{k}^{2}\right]\right\} \leq \mathcal{P}_{\max }
$$

for a predefined constant $\mathcal{P}_{\max }$.

\section{The Plant Model}

We consider the general arrangement of feedback control over a communication channel depicted in Fig. 2.

We assume the plant is LTI, finite dimensional and strictly proper with transfer function $G(z)$

$$
Y_{k}=G(z) * U_{k},
$$

where $U_{k}, Y_{k}$ are, respectively, the input and output of the plant. In this technical note, we restrict attention to plants that satisfy the following assumption.

Assumption 1: The plant is finite dimensional LTI and can be factored as

$$
G(z)=\frac{1}{(z-\phi)} G_{s}(z),
$$

with $|\phi|>1$, and where both $G_{s}(z)$ and $G_{s}^{-1}(z)$ have all poles strictly within the unit circle, and are proper.

From Assumption 1 it follows that we can write

$$
Y_{k+1}=\phi Y_{k}+G_{s}(z) * U_{k}
$$

\section{The Objective}

We restrict attention to encoders and decoders that are a causal function of the available data, namely the plant input, $U_{k}$, is generated by a causal decoding, $U_{k}=\mathcal{D}\left\{R^{k}\right\}$, of the received signal and conversely, the sent signal, $S_{k}$, is a causal encoding, $S_{k}=\mathcal{C}\left\{Y^{k}\right\}$, of the plant output.

We seek encoders and decoders that stabilize the plant in the following sense. By stability, we mean that for any distribution of initial conditions with finite second moment, all random variables converge at an exponential rate to stationary distributions, with well defined second moments. As an immediate consequence, [9, Thm 8.6.6] implies $\lim _{k \rightarrow \infty}\left\{h\left(Y_{k}\right)\right\}<\infty$.

\section{E. Channel Capacity Required for Stabilization}

We first briefly review results on the channel capacity required for stabilization of a scalar unstable system. There are a number of results in the literature that cover closely related results. In [16] stabilization of a vector unstable system over a noise free digital channel is considered. This was modified in [17] to the case of an AWGN channel. More recently, generalizations to multiple feedback stabilization problems over a shared network have been analyzed in [19]. The following result is a minor variant of a simplification of [19, Lemma 3.3].

Lemma 1: Consider the scalar unstable plant, (11), subject to Assumption 1 . Then, causal encoders and decoders that stabilize the plant (in the sense described above) exist only if

$$
C_{F B} \geq \log |\phi| \text {. }
$$

Proof: An outline of the proof follows. Further details can be found in [19, Lemma 3.3]. Note from (12) that we have $Y_{k}=\phi^{k} Y_{0}+$

${ }^{2}$ Note that several other equivalent power constraints can be used, since as shown in [11], optimal encoding uses equal power at each time. 
$f\left(R^{k-1}\right)$ where $f(\cdot)$ denotes the causal operator representing the combined effects of decoding the received signal, and convolution with $G_{s}(z)$, on the output $Y_{k}$. Therefore

$$
\begin{aligned}
h\left(Y_{k}\right) & =h\left(\phi^{k} Y_{0}+f\left(R^{k-1}\right)\right) \\
& \geq h\left(\phi^{k} Y_{0}+f\left(R^{k-1}\right) \mid R^{k}\right) \\
& =k \log |\phi|+h\left(Y_{0} \mid R^{k}\right) .
\end{aligned}
$$

From (14), the definition of capacity as the supremal limiting mutual information rate, and the data processing inequality it follows that

$$
\begin{aligned}
C_{F B} & \geq \limsup _{k \rightarrow \infty} \frac{1}{k} I\left(Y_{0} ; R^{k}\right) \\
& =\limsup _{k \rightarrow \infty} \frac{1}{k}\left(h\left(Y_{0}\right)-h\left(Y_{0} \mid R^{k}\right)\right) \\
& \geq \log |\phi|+\limsup _{k \rightarrow \infty} \frac{\left(h\left(Y_{0}\right)-h\left(Y_{k}\right)\right)}{k} .
\end{aligned}
$$

The result follows since the last term in (15) is zero due to the assumptions of finite initial entropy and stability.

\section{MA1 CHANNEL CAPACITY With FEEDBACK}

We now give a minor variant of the main result of [11] on the feedback capacity of an MA1 Gaussian channel.

Lemma 2: Consider the MA1 ACGN channel, (7), (8) under the power constraint, (9). The feedback capacity, (2), of this channel is given by:

$$
C_{F B}=\log _{2} w_{0}
$$

where $w_{0}$ is the unique solution in the range $(1, \infty)$ to the quartic equation

$$
\frac{\mathcal{P}_{\max }}{\sigma^{2}}=\left(w^{2}-1\right)\left(\frac{1-|\alpha|}{w}\right)^{2} .
$$

Proof: We begin by rescaling the channel random variables so that the white noise variance is unity. In particular, let $\bar{S}_{k}=S_{k} / \sigma$, $\bar{R}_{k}=R_{k} / \sigma, \bar{V}_{k}=V_{k} / \sigma$ and $\bar{N}_{k}=N_{k} / \sigma$. Clearly these rescaled random variables satisfy

$$
\begin{aligned}
\bar{V}_{k} & =\bar{N}_{k}+\alpha \bar{N}_{k-1} \\
E\left[\bar{N}_{k}^{2}\right] & =1 \\
\bar{R}_{k} & =\bar{S}_{k}+\bar{N}_{k}
\end{aligned}
$$

and the power constraint (9) becomes

$$
\lim _{N \rightarrow \infty}\left\{\frac{1}{N} \sum_{k=1}^{N} E\left[\bar{S}_{k}^{2}\right]\right\} \leq \frac{\mathcal{P}_{\max }}{\sigma^{2}} .
$$

The result (16), (17) follows immediately from [11, Theorem 1] with power constraint $\mathcal{P}_{\max } / \sigma^{2}$ and $x_{0}$ in [11] replaced by $w_{0}^{-1}$.

As noted in [11], following the structure of [12] also explored in [13], there exists a first-order autoregressive filter relating $S_{k}$ to $N_{k}$, that generates optimal transmissions [11, (40)]

$$
S_{k}=\beta S_{k-1}+\gamma N_{k-1}
$$

where $\beta=-\operatorname{sgn}(\alpha) / w_{0}$ and

$$
\gamma:=\operatorname{sgn}(\alpha) \sqrt{\frac{\mathcal{P}_{\max }\left(1-\beta^{2}\right)}{\sigma^{2}}} .
$$

We shall return to this fact later in Remark 1.

In what follows, we show a relationship between these results and results that may be obtained by applying $\mathcal{H}_{2}$ optimal control theory to the problem of LTI minimal SNR stabilization as in [8].

\section{LiNEAR MinimAL SNR STABILIZATION}

Consider the plant model, $G(z)$, as in (11) and the noise model (8). Assume that we have the trivial identity encoder ${ }^{3}$ and a linear time invariant decoder, $\mathcal{D}\left\{R^{k}\right\}=C(z) * R_{k}$ with $C(z)$ a transfer function such that the closed-loop system is stable. Under the assumption of closed loop stability, the power in the channel input, $S_{k}$, may be computed, in the disturbance free case, as $\left\|H_{s n}\right\|_{\mathcal{H}_{2}}^{2} \sigma^{2}$ where $H_{s n}$ is defined to be the closed loop transfer function from $N_{k}$ to $S_{k}$. Therefore, the SNR (that is the ratio of the power of the sent signal $S_{k}$ to the noise power, $\left.E\left[N_{k}^{2}\right]\right)$ in this case is precisely $\left\|H_{s n}\right\|_{\mathcal{H}_{2}}^{2}$, and stabilization within the SNR limit demands that $\left\|H_{s n}\right\|_{\mathcal{H}_{2}}^{2} \leq \mathcal{P}_{\max } / \sigma^{2}$.

The authors of [8] present the solution to the problem of minimizing $\left\|H_{s n}\right\|_{\mathcal{H}_{2}}^{2}$ over the class of all stabilizing controllers for a general LTI plant over a general colored noise channel with memory. For the case of a plant that satisfies Assumption 1, the results of [8] may be specialized as below in Lemma 3. The result below is proven using spectral theory and $\mathcal{H}_{2}$ theory, though the results could also be derived using Linear Quadratic Gaussian control theory, or Nevanlinna-Pick interpolation theory [14].

Lemma 3: Consider an LTI plant subject to Assumption 1 with MA1 ACGN channel (7), (8). Then stabilization is possible by LTI feedback subject to the power constraint (9) if and only if

$$
\frac{\mathcal{P}_{\max }}{\sigma^{2}} \geq\left(\phi^{2}-1\right)\left(\frac{1+\alpha}{\phi}\right)^{2} .
$$

Proof: (See also [8]). The steady state variance of the output can be determined using spectral analysis as

$$
E\left[S_{k}^{2}\right]=\left\|H_{s n}\right\|_{\mathcal{H}_{2}}^{2} \sigma^{2},
$$

where $H_{s n}$ is defined to be the closed loop transfer function from $N_{k}$ to $S_{k}$. We now follow similar derivations to those in [20].

Note that stabilizability subject to the power constraint (9) is equivalent to stabilizability subject to the constraint $\left\|H_{s n}\right\|_{\mathcal{H}_{2}}^{2} \leq \mathcal{P}_{\max } / \sigma^{2}$. Analysis of the closed loop equations yields that $H_{s n}$ in (25) is given by

$$
H_{s n}(z)=H(z) \frac{C(z) G(z)}{1-C(z) G(z)}
$$

We then use the Youla parametrization of all stabilizing controllers. We first express the plant as a fraction of rational stable proper transfer functions

$$
G(z)=\frac{N(z)}{M(z)}=\frac{z^{-1} G_{s}(z)}{\left(1-\phi z^{-1}\right)} .
$$

The class of all stabilizing controllers is then given by

$$
C(z)=-\frac{(X(z)+Q(z) M(z))}{(Y(z)-Q(z) N(z))}
$$

${ }^{3}$ Note that in the case of linear time invariant encoding and decoding with channel noise only, the decoder, plant and encoder all commute, and it is no loss of generality to take the trivial decoder. 
where $Q(z)$ is a stable proper transfer function, $X(z)=\phi G_{s}^{-1}(z)$ and $Y(z)=1$. Using (26) the closed loop transfer function can be expressed as

$$
\begin{aligned}
H_{s n}(z) & =-H(z) X(z) N(z)-H(z) Q(z) M(z) N(z) \\
& =-\phi \frac{1}{z} H(z)-H(z)\left(1-\phi \frac{1}{z}\right) \frac{Q^{\prime}(z)}{z}
\end{aligned}
$$

where $Q^{\prime}(z)=G_{s}(z) Q(z)$. In view of (29), and since $G_{s}^{-1} \in \mathcal{H}_{2}$, the problem of minimizing the transmitted power subject to stabilization of the closed loop is therefore equivalent to

$$
\begin{aligned}
& \min _{\{C \text { stabilizing }\}}\left\|H_{s n}(z)\right\|_{\mathcal{H}_{2}}^{2} \\
& \quad=\min _{\left\{Q^{\prime} \in \mathcal{H}_{2}\right\}}\left\|\phi \frac{1}{z} H(z)+H(z)\left(1-\phi \frac{1}{z}\right) \frac{Q^{\prime}(z)}{z}\right\|_{\mathcal{H}_{2}}^{2} .
\end{aligned}
$$

The factor $z^{-1}$ in (30) is all-pass and may be removed. Then apart from the term involving $\left(1-\phi z^{-1}\right)$, which is not stably invertible, the expression in (30) would be zero. We therefore proceed by extracting an all pass factor $\left(1-\phi z^{-1} / z^{-1}-\phi\right)$ as follows:

$$
\begin{aligned}
& \min _{\left\{Q^{\prime} \in \mathcal{H}_{2}\right\}}\left\|\phi H(z)+H(z)\left(1-\phi z^{-1}\right) Q^{\prime}(z)\right\|_{\mathcal{H}_{2}}^{2} \\
& =\min _{\left\{Q^{\prime} \in \mathcal{H}_{2}\right\}}\left\|\left(\frac{\phi(1-\phi z)}{(z-\phi)}\right) H(z)+H(z)\left(\frac{1}{z}-\phi\right) Q^{\prime}(z)\right\|_{\mathcal{L}_{2}}^{2}
\end{aligned}
$$

where $\mathcal{L}_{2}, \mathcal{H}_{2}$ and $\mathcal{H}_{2}^{\perp}$ are as defined in (4)-(6). We define $\Gamma(z):=$ $-\left(\alpha z^{-1}+\left(1+\alpha \phi^{-1}-\alpha \phi\right)\right)$ and

$$
r=\left(1-\phi^{2}\right)\left(\frac{1+\alpha}{\phi}\right) .
$$

We then further decompose (31) into components in $\mathcal{H}_{2}$ and $\mathcal{H}_{2}^{\perp}$ as $\min \left\|H_{s n}(z)\right\|_{\mathcal{H}_{2}}^{2}$

$=\min _{\left\{Q^{\prime} \in \mathcal{H}_{2}\right\}}\left\|\frac{r z}{(z-\phi)}+\left(\Gamma(z)+\frac{(z+\alpha)}{z} \frac{(1-\phi z)}{z} Q^{\prime}(z)\right)\right\|_{\mathcal{L}_{2}}^{2}$

$=\left\|\frac{r z}{(z-\phi)}\right\|_{\mathcal{H}_{2}^{\perp}}^{2}+\min _{\left\{Q^{\prime} \in \mathcal{H}_{2}\right\}}\left\|\left(\Gamma(z)+\frac{(z+\alpha)}{z} \frac{(1-\phi z)}{z} Q^{\prime}(z)\right)\right\|_{\mathcal{H}_{2}}^{2}$

$=\frac{r^{2}}{\phi^{2}-1}+0$.

Note that the last equality in (33) follows by taking $Q^{\prime}(z)=$ $-\Gamma(z) 1 /\left(1+\alpha z^{-1}\right)\left(z^{-1}-\phi\right)$. The result follows directly by substituting (32) in (33).

We now proceed to a result that follows in the case where $\alpha$ and $\phi$ have opposite signs. We later consider the case where $\alpha$ and $\phi$ have the same sign, in which a particular type of linear time varying encoding and decoding will be used.

Proposition 1: Consider the plant (10) with MA1 Gaussian channel (7). Suppose also that $\alpha$ and $\phi$ have opposite signs. Then stabilization is possible by LTI feedback subject to the power constraint (9) if and only if the channel capacity as described in Lemma 2 satisfies

$$
C_{F B} \geq \log _{2}|\phi| \text {. }
$$

Furthermore, if the constraint (34) is satisfied, then an exponentially stabilizing LTI controller that achieves the power constraint is given by the trivial encoding, $S_{k}=Y_{k}$, together with the decoder $U_{k}=$ $C(z)^{*} R_{k}$ where

$$
\frac{C(z)=-\left(\phi^{2}-1\right)(\phi+\alpha) z}{\left(\phi^{2} z+\alpha\right) G_{s}^{-1}(z) .}
$$

Proof: From Lemma 3, and the assumption on the signs of $\alpha$ and $\phi$ it follows that the plant is stabilizable by LTI feedback subject to the power constraint if and only if

$$
P_{\max } \sigma^{2} \geq\left(|\phi|^{2}-1\right)\left(\frac{1-|\alpha|}{|\phi|}\right)^{2} .
$$

Note that the right hand side of (36) is a monotonically increasing function of $|\phi|$, and that replacing $|\phi|$ in (36) by $w_{0}$ as defined in Lemma 2 gives equality. Therefore, $|\phi| \leq w_{0}$ and (34) is equivalent to (36).

To prove the second part of the proposition, we perform spectral analysis of the closed loop system with the control chosen as in (35). The closed loop transfer function from the noise source, $N_{k}$, to the transmitted signal, $S_{k}$, is given by

$$
\begin{aligned}
H_{s n}(z) & =\frac{C(z) G(z)}{1-C(z) G(z)} H(z) \\
& =\frac{-\left(\phi^{2}-1\right)\left(\frac{1+\alpha}{\phi}\right)}{\frac{\phi}{\left(\frac{z-1}{\phi}\right)}}
\end{aligned}
$$

It follows from (37) that the closed loop is exponentially stable. Furthermore, the asymptotic variance of $S_{k}$ can be computed as

$$
\begin{aligned}
\lim _{N \rightarrow \infty}\left\{\frac{1}{N} \sum_{k=1}^{N} E\left[S_{k}^{2}\right]\right\} & =\left\|H_{s n}(z)\right\|_{\mathcal{H}_{2}}^{2} \sigma^{2} \\
& =\frac{\left(\phi^{2}-1\right)^{2}\left(\frac{1+\alpha}{\phi}\right)^{2}}{\phi^{2}}\left\|\frac{1}{\frac{z-1}{\phi}}\right\|_{\mathcal{H}_{2}}^{2} \sigma^{2} \\
& =\left(\phi^{2}-1\right)\left(\frac{1-|\alpha|}{|\phi|}\right)^{2} \sigma^{2}
\end{aligned}
$$

and clearly in view of (36) the power constraint is satisfied.

Remark 1: Note that in the case where we have equality in (35), then clearly $w_{0}=|\phi|$. Therefore following the discussions after (22) (see also [11]) and using the fact that in Proposition $1, \operatorname{sgn}(\alpha)=-\operatorname{sgn}\left(w_{0}\right)$ we have $\beta=1 / \phi$. Also, in this case, from (23) it follows that

$$
\begin{aligned}
\gamma & =-\operatorname{sgn}(\phi) \sqrt{\left(\phi^{2}-1\right)\left(\frac{1+\alpha}{\phi}\right)^{2}\left(1-\beta^{2}\right)} \\
& =-\operatorname{sgn}(\phi) \sqrt{\frac{\left(\phi^{2}-1\right)^{2}\left(\frac{1+\alpha}{\phi}\right)^{2}}{\phi^{2}}} \\
& =\frac{-\left(\phi^{2}-1\right)\left(\frac{1+\alpha}{\phi}\right)}{\phi} .
\end{aligned}
$$

Therefore in the case of equality in (34), the controller (35) generates the same relationship between $S_{k}$ and $N_{k}$ as the filter in (22).

We now turn to the slightly more complicated case where $\alpha$ and $\phi$ have the same sign. In this case we utilize linear time varying operations as indicated in the following proposition.

Proposition 2: Suppose that the MA1 channel (7) has feedback capacity as defined in Lemma 2 that satisfies $C_{F B} \geq \log _{2}|\phi|$, and suppose also that $\alpha$ and $\phi$ have the same sign. Then the LTV feedback law

$$
\begin{aligned}
S_{k} & =(-1)^{k} Y_{k} \\
U_{k} & =\tilde{C}(z) *\left((-1)^{k} R_{k}\right) \\
\tilde{C}(z) & =-\frac{\left(\phi^{2}-1\right)(\phi-\alpha) z}{\left(\phi^{2} z-\alpha\right)} G_{s}^{-1}(z)
\end{aligned}
$$



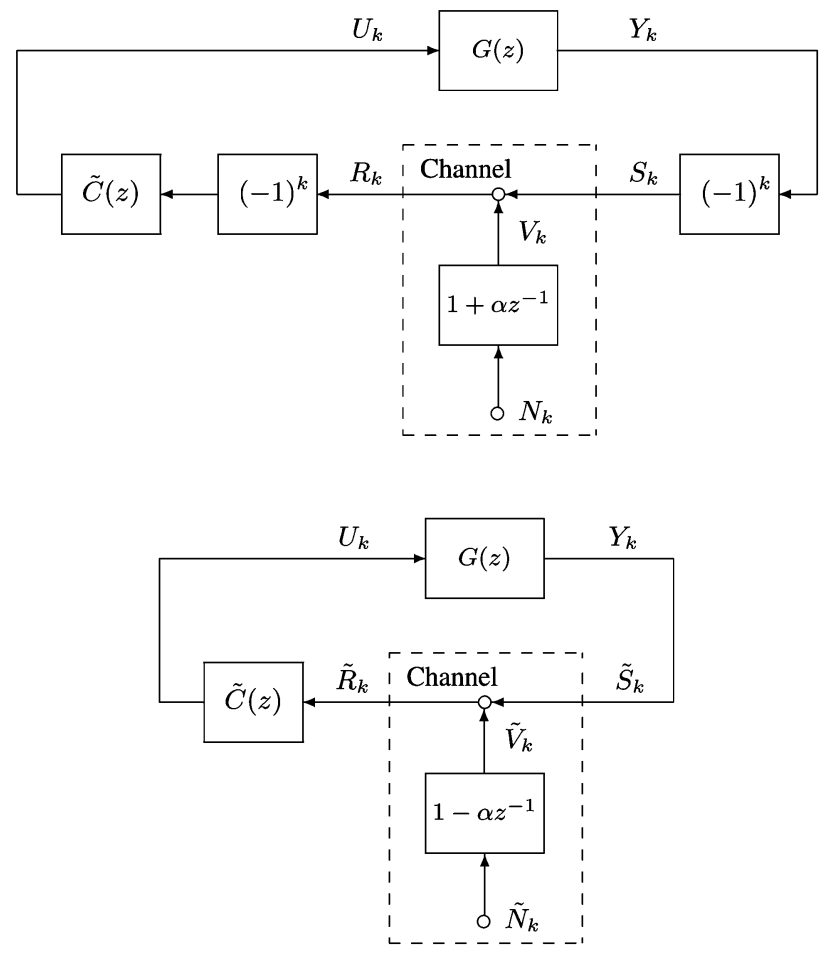

Fig. 3. Original LTV scheme (top) and equivalent interpretation (bottom).

exponentially stabilizes the plant with average transmitted power $\lim _{N \rightarrow \infty}\left\{1 / N \sum_{k=1}^{N} E\left[Y_{k}^{2}\right]\right\} \leq \mathcal{P}_{\max }$.

Proof: We first define transformed random variables $\tilde{R}_{k}=$ $(-1)^{k} R_{k}, \tilde{S}_{k}=(-1)^{k} S_{k}, \tilde{V}_{k}=(-1)^{k} V_{k}$, and $\tilde{N}_{k}=(-1)^{k} N_{k}$. Then using these definitions it is straightforward to show that

$$
\begin{aligned}
\tilde{R}_{k} & =\tilde{S}_{k}+\tilde{V}_{k} \\
\tilde{V}_{k} & =\tilde{N}_{k}-\alpha \tilde{N}_{k-1}
\end{aligned}
$$

where $\tilde{N}_{k}$ is a sequence of IID random variables with variance $\sigma^{2}$, and $E\left[\tilde{S}_{k}^{2}\right]=E\left[S_{k}^{2}\right]$. This transformation of the LTV scheme is illustrated in Fig. 3.

In addition, clearly the encoder and decoder in (38) can be expressed as

$$
\begin{aligned}
\tilde{S}_{k} & =Y_{k} \\
U_{k} & =\tilde{C}(z) * \tilde{R}_{k}
\end{aligned}
$$

and the remainder of the proof mirrors the proof of Proposition 1 except with the sign of $\alpha$ reversed.

\section{CONCLUSION}

In this technical note we have considered the problem of stabilization of a plant while simultaneously satisfying a channel SNR constraint. In particular, we examine MA1 colored Gaussian channels; and plants with a single unstable pole at $z=\phi ;|\phi|>1$, that are otherwise minimum phase and relative degree 1 . Using slight variants of existing results, we prove that stabilization is possible only if $C_{F B} \geq \log _{2}|\phi|$.
If this condition is satisfied we are able to exhibit linear coders and decoders that achieve stabilization subject to the SNR constraint.

For the LTI case, the results on the minimal channel SNR required to achieve stability generalize in a straightforward manner to more general plant descriptions and channel colorings. However, in these cases it is unclear whether nonlinear encoding and decoding may permit stabilization with a lower SNR than that achievable by the simpler linear schemes. Further research is needed to examine higher order plants, more complex channel codings, and the effect of stochastic plant disturbances on the results presented here.

\section{REFERENCES}

[1] A. J. Rojas, J. H. Braslavsky, and R. H. Middleton, "Feedback control over signal to noise ratio constrained communication channels," in Proc. SIAM Conf. Control Appl., San Francisco, CA, Jun. 2007, [CD ROM].

[2] P. Antsaklis and J. Baillieul, "Special issue on networked control," IEEE Trans. Automat. Control, vol. 49, no. 9, pp. 1421-1423, Sep. 2004.

[3] R. Bansal and T. Başar, "Simultaneous design of measurement and control strategies for stochastic systems with feedback," Automatica, vol. 25, no. 5, pp. 679-694, 1989.

[4] A. S. Matveev and A. V. Savkin, "The problem of lqg optimal control via a limited capacity communication channel," Syst. Control Lett., vol. 53, no. 1, pp. 51-64, 2004.

[5] J. H. Braslavsky, R. H. Middleton, and J. S. Freudenberg, "Feedback stabilization over signal to noise ratio constrained channels," IEEE Trans. Automat. Control, vol. 52, no. 8, pp. 1391-1403, Aug. 2007.

[6] A. Rantzer, "A separation principle for distributed control," in Proc. 45th IEEE Conf. Decision Control, San Diego, CA, Dec. 2006, pp. 3609-3613.

[7] N. C. Martins and T. Weissman, "Coding for additive white noise channels with feedback corrupted by quantization or bounded noise," IEEE Trans. Inform. Theory, vol. 54, no. 9, pp. 4274-4282, Sep. 2008.

[8] A. J. Rojas, J. H. Braslavsky, and R. H. Middleton, "Output feedback stabilization over bandwidth limited, signal to noise ratio constrained communication channels," in Proc. American Control Conf., Minneapolis, MN, Jun. 2006, pp. 2789-2794.

[9] T. M. Cover and J. A. Thomas, Elements of Information Theory, 2nd ed. New York: Wiley, 2006.

[10] T. M. Cover and S. Pombra, "Gaussian feedback capacity," IEEE Trans. Inform. Theory, vol. IT-35, no. 1, pp. 37-43, Jan. 1989.

[11] Y.-H. Kim, "Feedback capacity of the first-order moving average gaussian channel," IEEE Trans. Inform. Theory, vol. 52, no. 7, pp. 3063-3079, Jul. 2006.

[12] J. P. M. Schalkwijk and T. Kailath, "A coding scheme for additive noise channels with feedback part i: no bandwidth constraint," IEEE Trans. Inform. Theory, vol. IT-12, no. 2, pp. 172-182, Apr. 1966.

[13] S. A. Butman, "Linear feedback rate bounds for regressive channels," IEEE Trans. Inform. Theory, vol. IT-22, no. 3, pp. 363-366, May 1976.

[14] N. Elia, "When bode meets Shannon: Control-oriented feedback communication schemes," IEEE Trans. Automat. Control, vol. 49, no. 9, pp. 1477-1488, Sep. 2004.

[15] S. Yan, A. Kavčić, and S. Tatikonda, "On the feedback capacity of power-constrained gaussian noise channels with memory," IEEE Trans. Inform. Theory, vol. 53, no. 3, pp. 929-954, Mar. 2007.

[16] G. N. Nair and R. E. Evans, "Exponential stabilizability of finite dimensional linear systems with limited data rates," Automatica, vol. 39, no. 4, pp. 585-593, 2003.

[17] J. S. Freudenberg, R. H. Middleton, and V. Solo, "The minimal signal-to-noise ratio required to stabilize over a noisy channel," in Proc. Amer. Control Conf., Minneapolis, Mn, Jun. 2006, pp. 650-655.

[18] S. Tatikonda and S. Mitter, "Control over noisy channels," IEEE Trans. Automat. Control, vol. 49, no. 7, pp. 1196-1201, Jul. 2004.

[19] S. Yüksel and T. Başar, "Optimal signalling policies for decentralized multicontroller stabilizability over communication channels," IEEE Trans. Automat. Control, vol. 52, no. 10, pp. 1969-1974, Oct. 2007.

[20] O. Toker, J. Chen, and L. Qiu, "Tracking performance limitations in LTI multivariable systems," IEEE Trans. Circuits Syst. I, vol. 49, no. 5, pp. 657-670, May 2002. 